\title{
Estrategias de alimentación, evaluación del impacto ambiental y valoración económica de dietas de porcino ecológico
}

\author{
Argemí-Armengol, I. ${ }^{\circledR}$; Villalba, D.; Tor, M. y Álvarez-Rodríguez, J.
}

Departament de Ciència Animal. Universitat de Lleida. España.

\section{PALABRAS CLAVE}

Porcino.

Ecológico.

Cultivos locales.

Valor nutritivo.

Análisis ciclo de vida.

\section{ADDITIONAL KEYWORDS \\ Swine. \\ Organic. \\ Local crops.}

Nutritive value.

Life cycle assessment.

\section{INFORMATION}

\section{Cronología del artículo.}

Recibido/Received: 18.05 .2019

Aceptado/Accepted: 28.03.2020

On-line: 15.0.2020

Correspondencia a los autores/Contact e-mail:

immaargemi@gmail.com

\section{RESUMEN}

El principal desafío en alimentación para porcino ecológico es garantizar que los alimentos de la dieta cumplan los requerimientos nutricionales, en particular en proteína y perfil de aminoácidos, al finalizar la moratoria de uso de hasta un $5 \%$ de alimentos no ecológicos en las raciones, prevista para 2020. Así, este trabajo se planteó para proponer una estrategia de alimentación y analizar la viabilidad nutricional, económica y ambiental de formular raciones con alimentos alternativos, producidos localmente. Se seleccionó una muestra representativa de productos de cultivos ecológicos, de acuerdo al Reglamento (CE) 834/2007 y 889/2008, de fábricas de piensos y productores de Cataluña y zona próxima: cereales $(n=22)$, subproductos de cereales $(n=4)$ y concentrados de proteína vegetal $(n=25)$. Las dietas se formularon para las diferentes fases fisiológicas, ajustadas a las recomendaciones nutricionales españolas (FEDNA, convencional) y francesas (ITAB, ecológicas). Se formularon dietas prescindiendo de la soja (torta de presión y soja integral) según recomendaciones nutricionales francesas y utilizando fuentes proteicas adaptadas al clima mediterráneo (alverión, habas, guisantes y alholva) y cereales de invierno (centeno, trigo, cebada y triticale). Los impactos ambientales de las dietas (por unidad de pienso compuesto producido) en cuanto a demanda de fósforo, uso de energía no renovable, eutrofización, acidificación, cambio climático y ocupación de tierra, evaluados mediante el análisis de ciclo de vida de la base de datos francesa ECOALIM (2016), resultan mayores en lechones y menores en cerdas gestantes, y notablemente superiores si se formula según FEDNA vs. ITAB. En la valoración económica de las dietas (compra frente a autoproducción), resultaría más rentable un modelo de negocio con integración agropecuaria.

\section{Feeding strategies, environmental impact assessment and economic valuation of organic swine diets}

\section{SUMMARY}

The main dietary challenge for organic swine feeding is ensuring that feed fulfils the nutrient requirements, in particular to protein and amino acid profile, after the expiration that allow producers to include up to $5 \%$ non-organic feed within their rations, which is expected to 2020 . Thus, this work was designed to propose an organic feeding strategy and to analyse the nutritional, economic and environmental viability of locally produced feedstuff use. A representative sample of organic feed ingredients were selected, according to EC No 834/2007 and No 889/2008, in feed mills and producers from Catalonia and nearby area: cereals $(n=22)$, cereal by-products $(n=4)$ and protein concentrate $(n=25)$. Diets were formulated for the different physiological phases, adjusted to the Spanish (FEDNA, conventional) and French (TAB, organic) nutritional recommendations. Diets were formulated without soybean expeller or fullfat soybean when using French standards, using instead other protein sources adapted to the Mediterranean climate (Narbonne vetch, beans, peas, and fenugreek) and winter cereals (rye, wheat, barley and triticale). The assessment of environmental impacts (phosphorus demand, non-renewable energy, eutrophication, acidification, climate change and land occupation) of the diets (per unit of produced feed), through lifecycle analysis by means of the database ECOALM (2016), was higher in piglets and lower in pregnant sows, and much higher when formulating according to FEDNA vs. ITAB standards. In the economic valuation of the diets (purchase or self-production), a business model that integrates agriculture and livestock farming would be more profitable.

\section{INTRODUCCIÓN}

La formulación de dietas en porcino ecológico es más compleja que en convencional, ya que la ausencia de disponibilidad de materias primas encarece su coste y existen especificaciones de uso de ciertos ingredientes que difieren de la alimentación porcina convencional. Las diferencias más importantes, además de una procedencia de producción agraria ecológica, son: la necesidad de ofrecer forraje a los animales (pasto, heno, ensilado o paja), la prohibición de utilizar aminoácidos sintéticos (que puede condicionar la cobertura de las necesidades de aminoácidos esenciales si el nivel de proteína bruta de la dieta no es elevado), la prohibición de utilizar ingredientes provenientes de cultivos modificados genéticamente y la necesidad de utilizar tortas de oleaginosas procedentes de extracción mecánica (presión), de acuerdo con los Reglamentos (CE) 
834/2007 (DOUE, 2007) y 889/2008 (DOUE, 2008). Según el Reglamento de ejecución 505/2012 (DOUE, 2012), que modifica el artículo 19 del Reglamento (CE) $889 / 2008$, en el caso de cerdos y aves, al menos el $20 \%$ de la alimentación debería producirse en la misma zona, con el objetivo de fomentar la sostenibilidad productiva, integrando agricultura y ganadería. A partir del 1 enero de 2021, el nuevo Reglamento de producción ecológica, Reglamento (CE) 848/2018, será aún más restrictivo: incremento de alimento de la zona (de $20 \%$ a $30 \%$ ) y la utilización del $100 \%$ de materias ecológicas en monogástricos (excepción en lechones hasta $35 \mathrm{~kg}$ ) (DOUE, 2018a y b).

Según Calvar et al. (2010), la alimentación representa el $80 \%$ del coste de producción de la carne de cerdo ecológico. Por ello, la valoración de la autoproducción de materias puede tener un papel fundamental, siendo una práctica más establecida en granjas de rumiantes que en monogástricos. Aunque una parte de los ingredientes de la dieta proceden de procesos tecnológicos complejos (tortas de presión con o sin extrusión), otros ingredientes serían fáciles de producir y valorizar directamente en la granja.

El valor nutritivo de las materias primas ecológicas utilizadas en alimentación animal puede diferenciarse de las convencionales, dado que en agricultura ecológica no se utiliza abono mineral, limitando en algún caso el contenido de proteína bruta en las materias primeras ecológicas (Berry et al., 2002; Tejido et al., 2011). El aporte de forraje es obligatorio, y su potencial nutritivo puede contribuir a las necesidades de nutrientes y energéticas en los monogástricos (Rivera et al, 2001; Edwards, 2003; Früh, 2016; Wüstholz et al., 2017), aunque no se ha valorado suficientemente. Además, existe una falta de información sobre evaluaciones económicas de diferentes estrategias de producción de alimentos en las granjas de porcino.

El objetivo del presente trabajo es proponer dietas para porcino ecológico en las diferentes fases fisiológicas (gestación, lactación, lechones, crecimiento y acabado), eligiendo preferentemente materias primas locales, prescindiendo inicialmente de la soja y sus derivados, y minimizando el número de materias primas de la fórmula para poder aplicarse fácilmente en granja. Para las diferentes dietas también se evaluará el impacto ambiental de las mismas y se realizará un estudio económico comparando la autonomía de producción de la ración en comparación con la compra del pienso compuesto en una fábrica de piensos.

\section{MATERIAL Y MÉTODOS}

\section{VALOR NUTRITIVO DE INGREDIENTES}

Se utilizó una muestra representativa de productos de cultivos ecológicos, producidos en Cataluña y comunidades próximas, tales como cereales $(n=22)$, subproductos de cereales $(n=4)$, y concentrados de proteína vegetal $(n=25)$, procedentes de operadores certificados por los correspondientes Consejos de Producción Agraria Ecológica. Se determinó la composición química de cada uno de los ingredientes para estimar el valor nutritivo de los mismos. Las muestras se molturaron (diámetro $1 \mathrm{~mm}$ ) y se analizaron los siguientes parámetros por duplicado: materia seca (MS), cenizas, extracto etéreo con hidrólisis previa (EE), almidón, proteína bruta ( $\mathrm{N}$ x 6,25, PB), lisina (Lys), fibras ácido y neutro-detergente (FAD y FND) con adición de amilasa previa, fibra bruta (FB) calculada a partir de la FAD (INRA, 2018) y macrominerales (calcio y fósforo), siguiendo las metodologías de AOAC (2000). Se determinó el contenido de lisina (Lys) de las materias primas por UPLC-SRM previa hidrolisis en medio ácido (Álvarez-Rodríguez et al., 2018).

La predicción de la energía neta (EN) de los ingredientes se realizó con las ecuaciones recogidas en el Manual "EvaPig®" (Noblet et al., 2008), diferenciando entre adultos (ENa) y crecimiento (ENg), con el proceso siguiente:

-Energía bruta (EB, MJ / kg MS): $\mathrm{EB}=17,57+0,0535$ $\mathrm{x} \mathrm{PB}+0,2168 \times \mathrm{EE}+0,0284 \times \mathrm{FB}-0,1861 \times$ cenizas .

-Energía digestible (ED, MJ/kg MS), se calcula diferenciando para la fase de crecimiento (EDg) y adulto (EDa):

$$
\mathrm{EDg}=\mathrm{EB} \times \mathrm{Edg} .
$$

Digestibilidad de la energía en crecimiento (Edg, \%) $=90,1-1,57 \times$ FB. 100).

$\mathrm{EDa}=\mathrm{EDg}+4,2 \times(1-$ cenizas $/ 100) \times(1-\mathrm{MOd} /$

Digestibilidad de la materia orgánica $(\mathrm{MOd}, \%)=$ 7,45 + 0,949 x EDg -0,04x PB.

-Energia neta (EN, MJ/kg MS) (misma ecuación para crecimiento y adultos, utilizando EDa ò EDg):

$\mathrm{ENa}$ o $\mathrm{ENg}=0,703 \times \mathrm{EDa}$ ò $\mathrm{g}-0,0404 \times \mathrm{PB}+0,0662$ $x$ EE $+0,0197 x$ almidón $-0,0409 x$ FB.

Donde la PB, EE, FB, almidón y cenizas están expresados en porcentaje (sobre MS).

Se determinó, además, el perfil de ácidos grasos de los ingredientes por cromatografía gas-líquido en columna capilar (SP2330; Supelco, Tres Cantos, Madrid, España) y detector tipo FID (Tor et al., 2015).

\section{FORMULACIÓN DE DIETAS}

Se creó una base de datos de diferentes ingredientes $(\mathrm{n}=27)$ que incluía cereales (cebada, centeno, avena, maíz, triticale, trigo), concentrados de proteína vegetal (alverjón, alholva, guisante de primavera, habas, yeros, soja entera, semilla de girasol, torta de girasol, torta de soja), subproductos de cereales (germen de maíz, harinilla de maíz, salvado de trigo), alimentos fibrosos (cascarilla de espelta, paja de cereal, granulado de alfalfa), aceite de soja y complementos vitamínicominerales (carbonato cálcico, fosfato bicálcico, cloruro sódico, corrector vitamínico-mineral).

Se formularon piensos adaptados a cada fase fisiológica (lechones, cerdos en crecimiento-cebo y cerdas reproductoras en lactación y gestación), según las recomendaciones nutricionales para cerdo convencional de la Fundación Española para el Desarrollo de la Nutrición Animal-FEDNA (2013) y para cerdo ecológico, según las recomendaciones conjuntas del instituto fran- 
cés Institut Technique de l'Agriculture Biologique-ITAB e IFIP-Institut $d u$ porc (2014). Únicamente se ha tenido en cuenta las necesidades de proteína bruta y de lisina para garantizar las necesidades de aminoácidos, pero no ha sido posible considerar el concepto de proteína ideal, ya que no se disponía de resultados analíticos de composición de todos los aminoácidos esenciales.

Para establecer los límites máximos de incorporación de ingredientes, se consideraron los factores antinutritivos de éstos y su limitación en las raciones, tomando las recomendaciones de FEDNA e ITAB, respectivamente. En el alverjón, la alholva y los yeros no fue posible encontrar una referencia para su límite de incorporación en pienso, por lo que se tomaron los límites de los ingredientes más próximos de la misma familia (leguminosas) que ofrecían ambas instituciones, así como los estudios de Gómez-Izquierdo (2015) y Martín-Pedrosa et al. (2016).

Las fórmulas de pienso se resolvieron con un método de optimización lineal mediante el programa Winfeed ${ }^{\circledR}$ (Universidad de Cambridge, Cambridge, Reino Unido). En el caso que la solución de la fórmula mostrara ingredientes con un nivel de inclusión $<2 \%$, se han eliminado del inventario y se han reformulado nuevamente para obtener una nueva solución. Asimismo, se ha limitado la inclusión del número de cereales en cada fórmula, para facilitar su fabricación en granja. Para ello, se han considerado los dos cereales con mayor proporción en la solución de la fórmula, se han eliminado del inventario el resto y se ha reformulado nuevamente. En cuanto al concentrado de proteína vegetal, se ha procurado prescindir de la soja y sus derivados, y cuando no ha sido posible alcanzar los requerimientos recomendados, se ha introducido en el inventario. Se han tomado como límites de incorporación en la dieta los valores de diferentes ensayos de estrategias de formulación con resultados favorables, tanto en rendimientos productivos como bienestar animal, en cerdos de engorde y reproductoras, dónde utilizaban forrajes hasta un 15-30\% en engorde y un 25$50 \%$ en gestantes (Edwards, 2003; ITAB, 2014; Jakobsen et al., 2014; Smith et al., 2014; Wüstholz et al., 2017). En todos los casos, se ha asumido un porcentaje constante de inclusión de corrector vitamínico-mineral del 0,4\%.

En una primera fase se han formulado dietas para todos los tipos fisiológicos, mientras que en una segunda fase, se ha considerado la estrategia de fabricar tres fórmulas base (lechones, cerdos en crecimiento y cerdas lactantes) y el resto, gestantes y de cerdos en acabado, se han formulado a partir de ellas e incluyendo además un porcentaje de forraje y/o alimento fibroso (granulado de alfalfa, cascarilla de espelta, paja de cereal).

\section{IMPACTO AMBIENTAL DE LAS DIETAS FORMULADAS}

Para la evaluación del impacto ambiental de las dietas formuladas se ha utilizado la base de datos ECOALIM, que incluye los ingredientes más utilizados en el contexto francés, así como su inventario de impactos asociados (Wilfart et al., 2016), en función del método y origen de producción y / o transformación. Se seleccionaron los ingredientes de esa base de datos más parecidos a la base de datos propia. Los seis impactos considerados fueron la demanda de fósforo (PC, en $\mathrm{kg}$ $\mathrm{P})$, la demanda de energía no renovable (CED, en $\mathrm{MJ})$, el potencial de cambio climático (GWP, en $\mathrm{kg} \mathrm{CO}_{2}$-eq), acidificación (AP, en molc $\mathrm{H}+-\mathrm{eq})$, eutrofización (EP, en kg P-eq), y ocupación de tierra (LO, en $\mathrm{m}^{2}$ ).

En la selección de los ingredientes de la base de datos ECOALIM se ha considerado: en los cereales, origen de agencia de almacenaje o fábrica de piensos, de producción y transformación a nivel nacional y abono mediante fertilizantes orgánicos; en el caso de leguminosas grano y girasol, cultivos nacionales y producidos directamente en campo; en el caso de harinas y subproductos de cereales, procedentes de molino nacional y origen en planta de transformación; en el caso de torta de presión de soja procedente de Estados Unidos, prensado nacional y en planta de transformación y finalmente en el caso de aceite de soja, procedente de Brasil, no asociado a deforestación, prensado nacional y planta de transformación.

En la evaluación del impacto ambiental de las dietas se han considerado los impactos por quilo de pienso producido, y según el consumo anual en las diferentes fases del ciclo productivo (1 cerda y su descendencia anual): número de partos por cerda y año (2), número de lechones por parto (10), días de gestación (114) y meses de crecimiento y engorde (7). La estimación del consumo de alimento por fase fisiológica y animal se ha considerado: el lechón ( $<30 \mathrm{~kg}$ de PV; ITAB, 2014) de $0,6 \mathrm{~kg} /$ día, el cerdo en crecimiento $(<60 \mathrm{~kg}$; ITAB, 2014) de $1,5 \mathrm{~kg} /$ día, el cerdo de engorde-acabado $(<110 \mathrm{~kg}$; ITAB, 2014) de 2,5 kg/día; en las gestantes de $2 \mathrm{~kg} /$ día y en lactantes de $5 \mathrm{~kg} /$ día. Las duraciones de cada fase fisiológica son: en lechones 84 días (correspondientes a 49 días de lactación y 35 días postdestete), cerdo en crecimiento 49 días y cerdo engorde-acabado 77 días; y las reproductoras de 225 días/año de gestación y 140 días/año en lactación (dos periodos de 49 días de lactación y 21 días de pre y postcubrición, total de 140 días).

\section{VALORACIÓN ECONÓMICA DEL COSTE DE LOS PIENSOS EN} DIFERENTES ESCENARIOS

Con las fórmulas de pienso obtenidas, se ha realizado una valoración económica, teniendo en cuenta dos escenarios: coste de compra en la fábrica de piensos vs. coste de autoproducción de las materias primas, calculado según el consumo anual de una cerda y su descendencia.

\section{COSTES DE COMPRA DEL PIENSO EN FÁBRICA DE LA ZONA}

Según el Consejo Catalán de Producción Agraria Ecológica (CCPAE), en fecha de octubre de 2018, existían 24 operadores que comercializaban materias primas o compuestos para la elaboración de piensos. Se ha recogido el coste medio de compra en fábricas de piensos de Cataluña, especializadas en producción ecológica, siendo: gestantes de $0,38 € / \mathrm{kg}$ (impuestos y transporte incluidos), lactante de $0,44 € / \mathrm{kg}$, lechón de $1,09 € / \mathrm{kg}$ y acabado de $0,44 € / \mathrm{kg}$.

\section{COSTES DE AUTOPRODUCCIÓN DE LA FÓRMULA COMPLETA}

En el cálculo del coste de autoproducción de las materias primas (cereales y leguminosas) se ha considerado el coste directo (alquiler de máquinas agrícolas 
y conductor) para producción de cultivos extensivos en secano, correspondiente a: trabajos primarios del suelo (abonado orgánico, laboreo con arada de discos, laboreo con chisel y laboreo con vibrocultor), trabajos secundarios (cultivador, arada de varillas flexible), cosecha y derivados (coste de siembra, coste de compra de semilla, cosechadora, segadora acondicionadora, rastrillar, picar, transporte y seguro), según el cultivo (Tabla I).

En la valoración se ha considerado el coste de producción del cultivo por familia más elevado, en cereales de $376 € /$ ha y en leguminosas de $464 € /$ ha. Se ha incluido el coste del seguro, el coste de la molienda, teniendo en cuenta como coste la amortización anual de $\mathrm{kg} / \mathrm{ha}$ en guisante (Pisum sativum), $1.500 \mathrm{~kg} / \mathrm{ha}$ en haba (Vicia fava var. Equina) y $1.700 \mathrm{~kg} / \mathrm{ha}$ en alverjón (Vicia narbonensis).

En los ingresos, se han considerado las posibles ayudas a agricultura ecológica, en base al cumplimiento de la condicionalidad que establece el artículo 92 del Reglamento (UE) 1306/2013. El cálculo se ha realizado de acuerdo al Plan de Desarrollo Rural de Cataluña para el período 2014-2020, dónde la ayuda a agricultura ecológica es de $145 € /$ ha (cereal secano) y la ayuda a ganadería ecológica es de $215 €$ / ha por cerda en ciclo cerrado y de $131 € /$ cerdo de engorde; con el requisito de tener como mínimo en la explotación la superficie agrícola de 0,30 ha/UGM en reproductoras y 0,23 ha/

Tabla I. Estimación de costes de semilla y maquinaria en agricultura ecológica (Estimating seed and machinery costs in organic farming).

\begin{tabular}{|c|c|c|c|c|c|c|}
\hline \multirow{2}{*}{\multicolumn{2}{|c|}{$\begin{array}{l}\text { Cultivos } \\
\text { Guisante }\end{array}$}} & \multicolumn{3}{|c|}{ Leguminosas $(€ / \mathrm{ha})$} & \multicolumn{2}{|c|}{ Cereales (€/ha) } \\
\hline & & \multirow[t]{2}{*}{ Haba } & \multirow[t]{2}{*}{ Alverjón, Alholva } & \multirow[t]{2}{*}{ Cebada, Centeno } & \multirow[t]{2}{*}{ Triticale } & \\
\hline \multicolumn{3}{|l|}{ Semilla } & & & & \\
\hline \multicolumn{2}{|c|}{ Coste de semilla $(€ / \mathrm{kg})$} & 0,50 & 0,38 & 1,85 & 0,35 & 0,45 \\
\hline \multicolumn{2}{|c|}{ Dosis de siembra $(\mathrm{kg} / \mathrm{ha})$} & 170 & 150 & 120 & 180 & 170 \\
\hline \multicolumn{2}{|l|}{ Coste $(€ /$ ha $)$} & 85 & 57 & 222 & 63 & 77 \\
\hline \multicolumn{7}{|l|}{ Trabajos maquinaria } \\
\hline \multirow{4}{*}{$\begin{array}{l}\text { Laboreo primario } \\
\text { suelo }\end{array}$} & Abonado (esparcir) & - & - & - & 38 & 38 \\
\hline & Arada discos & 28 & 28 & 28 & 28 & 28 \\
\hline & Chisel & 31 & 31 & 31 & 31 & 31 \\
\hline & Vibro cultor & 18 & 18 & 18 & - & - \\
\hline \multirow{3}{*}{$\begin{array}{l}\text { Laboreo secundario } \\
\text { suelo }\end{array}$} & Cultivador & 20 & 20 & 20 & - & - \\
\hline & Arada púas flexibles & - & - & - & 15 & 15 \\
\hline & Coste siembra & 34 & 34 & 34 & 34 & 34 \\
\hline \multirow{3}{*}{$\begin{array}{l}\text { Cosecha cultivos y } \\
\text { derivados }\end{array}$} & Recolección y picar & 90 & 90 & 90 & - & - \\
\hline & Recolección grano & - & - & - & 61 & 61 \\
\hline & Embalar & - & - & - & 30 & 30 \\
\hline \multirow{3}{*}{ Otros } & Transporte paja & - & - & - & 18 & 18 \\
\hline & Transporte grano & 11 & 11 & 11 & 11 & 11 \\
\hline & Seguro & 10 & 10 & 10 & 10 & 10 \\
\hline \multicolumn{2}{|l|}{ TOTAL, Costes (€/ha) } & 327 & 299 & 464 & 339 & 353 \\
\hline
\end{tabular}

los equipos de fabricación (silos, molino y mezcladora) y la energía eléctrica consumida $(30 € / T)$. En algunas materias primas ha sido necesario incluir su coste de compra: corrector vitamínico-mineral $(2,00 € / \mathrm{kg})$, granulado de alfalfa $(1,65 € / \mathrm{kg})$, salvado de trigo $(0,30 € /$ $\mathrm{kg})$, soja integral $(0,84 € / \mathrm{kg})$, y torta de presión de soja $(0,84 € / \mathrm{kg})$; según fábrica de piensos de referencia del año 2018 en Cataluña.

La referencia de rendimientos de los cultivos se ha obtenido a través de la Generalitat de Cataluña (Gencat, 2017) y de datos de entrevistas a diferentes agricultores de producción ecológica suministradores de las materias primas analizadas (cereales y leguminosas). Los rendimientos estimados fueron: $3.500 \mathrm{~kg} / \mathrm{ha}$ en cebada (Hordeum vulgare), $3.200 \mathrm{~kg} /$ ha en centeno (Secale cereale), $3.200 \mathrm{~kg} /$ ha en trigo (Triticum aestivum), 2.500
UGM en engorde, estableciendo un máximo subvencionable de 1,2 UGM/ha. Se ha tenido en cuenta un escenario de arrendamiento de la tierra de $176 € /$ ha (MAPA, 2016), dónde para alimentar a una cerda y su descendencia hace falta 2,5 ha/año, equivalente a 440 $€ /$ cerda.

\section{RESULTADOS Y DISCUSIÓN}

\section{VALOR NUTRITIVO DE LOS INGREDIENTES}

En la Tabla II, se representan los resultados del análisis químico de nutrientes de las materias primas, dónde se ha estimado el valor energético para cerdas adultas y cerdos en crecimiento utilizado para la for- 
mulación de piensos. Los ingredientes más concentrados energéticamente $(\mathrm{MJ} / \mathrm{kg})$, de mayor a menor, son el aceite de soja, la semilla de girasol, la soja entera, el grano de maíz y el trigo; y los menos concentrados energéticamente son las habas, torta de girasol, granulado de alfalfa, cascarilla de espelta y paja de cereal. Si se compara la diferencia del valor energético entre adultas y crecimiento, destaca en mayor grado la paja de cereal $(+2,02)$, cascarilla espelta $(+1,72)$, granulado alfalfa $(+1,45)$ y torta de presión de girasol $(+1,07)$; $y$ menos diferencia en aceite de soja $(+0,12)$, triticale y maíz $(+0,36)$ y trigo $(+0,37)$. En la I, se muestra el perfil de ácidos grasos de los ingredientes utilizados. En general, destaca la elevada ratio de ácidos grasos poliinsaturados/ácidos grasos saturados (AGPI/AGS) en la soja y sus derivados (torta de presión y aceite), el girasol entero y el maíz (así como algunos de sus derivados como las harinillas de maíz). Así mismo, es destacable la elevada ratio de ácidos grasos polinsaturados omega-6/ ácidos grasos polinsaturados omega-3 (AGPI n-6/AGPI n-3) en el caso de la semilla de girasol y su torta de presión, así como en los subproductos del maíz (harinillas y germen) y el maíz grano.

\section{FORMULACIÓN DE DIETAS}

\section{FORMULACIÓN SEGÚN REQUERIMIENTOS DE FEDNA E ITAB}

La propuesta de formulación de dietas de porcino reproductor (gestantes y lactantes) y engorde (lechones, crecimiento y acabado) a partir del valor nutritivo de los ingredientes ecológicos recogidos se resumen en la Tabla IV.

Comparando la EN (MJ/kg), PB (\%) y Lys (\%) de las dietas formuladas atendiendo a las necesidades propuestas por ITAB y FEDNA, se constata que en todas las fases fisiológicas son superiores en FEDNA. En engorde los requerimientos son hasta 18\% y 30\% superiores en ENg y Lys, respectivamente, y en la fase de reproductoras son hasta un 11\% y 19\% superiores en ENa y Lys. Se observa que la relación Lys/EN es superior en FEDNA que en ITAB en todas las fases (lechones: $7,4 \%$, crecimiento: $16,6 \%$, acabado: $8,9 \%$, y lactación: $13,9 \%)$, excepto en gestantes (-8,6\%). Con los alimentos analizados, ha sido posible formular dietas en todas las fases, según FEDNA e ITAB, excepto en lechones, por no llegar a cubrir las necesidades del primer aminoácido limitante en cerdo (Lys FEDNA=1,35\% y Lys ITAB $=1,1 \%$ ), resultando las fórmulas propuestas un $29 \%$ y un $25 \%$ deficientes en Lys, respectivamente.

Las dietas en base a los requerimientos de ITAB se han formulado prescindiendo del haba de soja y de la torta de soja, excepto en el pienso de lechones. En base a los requerimientos de FEDNA, sólo ha sido posible formular dietas sin soja en la fase de gestantes. A pesar de los objetivos iniciales, no se ha podido prescindir totalmente del haba de soja ya que su aporte de lisina se aproxima muy bien a los requerimientos, tanto de ITAB como FEDNA.

Las fórmulas planteadas se ven condicionadas por la mínima información existente acerca de los límites de incorporación de ciertas leguminosas poco caracterizadas para alimentación animal (alverjón, habas, yeros, alholva).
Para reducir el número de dietas en la granja, se ha propuesto la dieta de gestantes y acabado a partir de dos dietas núcleo de lactantes y crecimiento, respectivamente, ajustando las concentraciones de alimentos fibrosos (Tabla V). El uso de forrajes es obligatorio en producción ecológica, pero el potencial de éstos para contribuir a las necesidades nutricionales de los monogástricos no es muy claro y a menudo no se tiene en cuenta en el plan de alimentación ni en gestantes ni en la fase de engorde. En una dieta completa (unifeed) mezclando pienso (con ingredientes mayoritarios: triticale y guisante) y ensilado de alfalfa, en cerdos en crecimiento, no se encontraron diferencias significativas en el crecimiento en comparación con una dieta a base de únicamente pienso (Wüstholz et al., 2017), apoyando el hecho que los forrajes pueden contribuir en parte al aporte de aminoácidos (Edwards, 2003; Früh et al, 2014).

No obstante, la producción de forrajes en explotaciones de monogástricos no es habitual, y la disponibilidad de una gran oferta de piensos tampoco es operativa en casos de explotaciones de pequeño tamaño. Por ello, en cerdas gestantes sería posible utilizar como base el pienso de cerdas lactantes (75\%) y añadir como alimentos fibrosos un $20,7 \%$ de salvado de trigo y $4,9 \%$ de paja de cereal de invierno picada, mientras en acabado sería posible mezclar un $10 \%$ de salvado de trigo con un $90 \%$ de pienso de cerdos en crecimiento.

Evaluación impacto ambiental y evaluación económica de las dietas

La obtención de alimentos para la producción animal ocasiona un impacto ambiental asociado (importación de materias primas que compiten por la utilización de tierras para otros usos, consumo de combustibles fósiles, generación de gases de efecto invernadero, eutrofización, etc.). El análisis del ciclo de vida es la metodología más utilizada y reconocida para estimar el impacto de la producción animal (de Vries et al., 2010).

El impacto ambiental de las dietas está influenciado por los tipos de ingredientes utilizados, donde algunos de ellos son incorporados en un pequeño porcentaje, pero su impacto ambiental por $\mathrm{kg}$ es elevado, por ejemplo, los fosfatos minerales (Garcia-Launay et al., 2014). En la actualidad existe información del impacto ambiental evaluado por análisis del ciclo de vida del porcino expresado por unidad de peso vivo producido. Sin embargo, en esos trabajos, se asume una composición de pienso estándar para realizar los cálculos, por lo que no se tienen en cuenta variaciones en los ingredientes que componen la fórmula. Los impactos ambientales estudiados: PC (kg), CED (MJ), GWP (kg CO -eq), EP $\left(\mathrm{kg} \mathrm{PO}_{4}, \mathrm{AP}\left(\right.\right.$ molc $\mathrm{H}^{+}$-eq) y $\mathrm{LO}\left(\mathrm{m}^{2}\right)$, por tonelada de pienso de cada fórmula propuesta se representan en la Figura 1. Los impactos PC (kg P), CED (MJ) y AP (molc $\mathrm{H}+\mathrm{eq}$ ), por $\mathrm{T}$ de pienso, se observa que son superiores según recomendaciones FEDNA respecto a ITAB. Esto puede deberse a que FEDNA propone piensos más concentrados en nutrientes, que requieren la inclusión de ingredientes más ricos en proteína, que tienen una mayor demanda de recursos (energía, agua y nutrientes) durante su proceso de producción. Por el contrario, en el caso del GWP $(\mathrm{kg} \mathrm{CO} 2), \mathrm{LO}\left(\mathrm{m}^{2}\right)$ y el EP $(\mathrm{kg} \mathrm{PO})$ 
Tabla II. Valor nutritivo y energético de ingredientes ecológicos para formulación piensos (\% sobre materia fresca) (Nutritional and energy value of organic ingredients for feed formulation (\% on fresh matter)).

\begin{tabular}{|c|c|c|c|c|c|c|c|c|c|c|c|c|}
\hline Materia prima & $\mathrm{MS}(\%)$ & $\mathrm{PB}(\%)$ & $\mathrm{FB}(\%)$ & FND(\%) & $\mathrm{FAD}(\%)$ & $\mathrm{EE}(\%)$ & Almidón(\%) & Lys(\%) & $\mathrm{Ca}(\%)$ & $\begin{array}{l}P \\
(\%)\end{array}$ & $\begin{array}{c}\mathrm{EN}_{\mathrm{a}} \\
(\mathrm{MJ} / \\
\mathrm{kg})\end{array}$ & $\begin{array}{c}\mathrm{EN}_{\mathrm{g}} \\
(\mathrm{MJ} / \\
\mathrm{kg})\end{array}$ \\
\hline \multicolumn{13}{|l|}{ Cereales } \\
\hline Avena & 89,59 & 10,27 & 11,83 & 34,90 & 14,33 & 5,29 & 35,10 & 0,35 & 0,14 & 0,30 & 8,93 & 8,18 \\
\hline Cebada & 89,78 & 9,88 & 6,28 & 28,26 & 7,84 & 2,88 & 47,83 & 0,36 & 0,07 & 0,26 & 9,83 & 9,29 \\
\hline Centeno & 89,16 & 7,59 & 3,67 & 17,88 & 4,79 & 1,80 & 49,93 & 0,29 & 0,04 & 0,30 & 10,42 & 10,04 \\
\hline Maíz & 87,61 & 7,27 & 3,13 & 12,52 & 4,14 & 4,70 & 62,61 & 0,22 & 0,01 & 0,25 & 11,30 & 10,98 \\
\hline Trigo & 90,00 & 11,12 & 2,96 & 14,35 & 3,96 & 2,35 & 58,57 & 0,33 & 0,06 & 0,27 & 10,93 & 10,60 \\
\hline Triticale & 89,90 & 10,10 & 2,82 & 13,75 & 3,79 & 1,97 & 57,12 & 0,31 & 0,04 & 0,27 & 10,87 & 10,54 \\
\hline \multicolumn{13}{|c|}{ Subproductos de cereales } \\
\hline Germen maíz & 90,33 & 15,55 & 12,44 & 27,50 & 15,04 & 22,61 & 22,07 & 0,56 & 0,07 & 1,29 & 11,53 & 10,84 \\
\hline Harinilla maíz & 86,48 & 8,63 & 3,34 & 18,34 & 4,38 & 7,04 & 50,87 & 0,29 & ND & 0,48 & 11,05 & 10,68 \\
\hline Salvado trigo & 88,47 & 15,00 & 7,88 & 30,79 & 9,70 & 4,08 & 30,35 & 0,50 & 0,06 & 0,66 & 9,10 & 8,50 \\
\hline \multicolumn{13}{|c|}{ Concentrados de proteína vegetal } \\
\hline Girasol & 94,00 & 16,27 & 18,10 & 30,92 & 21,67 & 44,89 & 1,99 & 0,43 & 0,21 & 0,34 & 14,44 & 13,53 \\
\hline Guisantes & 87,99 & 20,26 & 7,15 & 18,14 & 8,84 & 1,82 & 45,49 & 1,30 & 0,26 & 0,31 & 9,45 & 8,96 \\
\hline Alverjón & 88,10 & 26,98 & 10,62 & 17,70 & 12,90 & 2,20 & 37,10 & 1,43 & 0,08 & 0,19 & 8,88 & 8,28 \\
\hline Alholva & 90,10 & 27,28 & 8,73 & 33,60 & 10,70 & 9,30 & 14,60 & 1,32 & 0,79 & 0,27 & 9,58 & 8,91 \\
\hline Habas & 89,22 & 22,88 & 12,05 & 19,01 & 14,57 & 1,91 & 11,41 & 1,74 & 0,14 & 0,39 & 8,17 & 7,53 \\
\hline Soja entera & 87,36 & 29,54 & 6,77 & 11,92 & 8,40 & 17,25 & 4,65 & 1,72 & ND & ND & 11,44 & 11,01 \\
\hline $\begin{array}{l}\text { Torta } \\
\text { presión girasol }\end{array}$ & 93,48 & 29,70 & 19,71 & 37,93 & 23,55 & 7,81 & 3,95 & 1,06 & 0,47 & 0,83 & 7,45 & 6,45 \\
\hline $\begin{array}{l}\text { Torta de presión } \\
\text { soja }\end{array}$ & 92,61 & 42,94 & 7,96 & 18,37 & 9,82 & 7,72 & 9,17 & 2,01 & 0,25 & 0,54 & 9,66 & 9,13 \\
\hline Yeros & 90,07 & 16,37 & 9,42 & 19,71 & 11,50 & 2,55 & 39,97 & 0,89 & 0,60 & 0,18 & 9,40 & 8,83 \\
\hline \multicolumn{13}{|c|}{ Ingredientes fibrosos } \\
\hline Cascarilla espelta & 91,53 & 5,16 & 32,64 & 68,90 & 38,64 & 1,44 & 7,25 & 0,14 & 0,21 & 0,16 & 4,04 & 2,47 \\
\hline Granulado alfalfa & 91,77 & 11,91 & 32,24 & 51,35 & 38,18 & 2,11 & - & 0,31 & 1,49 & 0,17 & 4,08 & 2,75 \\
\hline Paja de cereal & 91,85 & 2,97 & 41,33 & 73,52 & 48,80 & 1,38 & 2,78 & 0,09 & 0,24 & 0,06 & 2,74 & 0,88 \\
\hline \multicolumn{13}{|l|}{ Aceites } \\
\hline Aceite soja & 99,82 & - & - & - & - & 99,44 & - & - & - & - & 32,27 & 32,15 \\
\hline
\end{tabular}

las dietas de lechones y crecimiento tienen más impacto según recomendaciones del Instituto francés (ITAB).

Las dietas formuladas con mayor impacto de PC son las de cerdas lactantes y lechones en base a FEDNA (9,03 y $8,55 \mathrm{~kg} \mathrm{P} / \mathrm{T}$, respectivamente) y lechones según $\operatorname{ITAB}(8,13 \mathrm{~kg} \mathrm{P} / \mathrm{T})$ y menor la de cerdas gestantes $(3,78$ $\mathrm{kg} \mathrm{P} / \mathrm{T})$. Valores inferiores fueron encontrados por Garcia-Launay et al. (2016), dónde el impacto de una dieta estándar mixta ( $40 \%$ crecimiento / $60 \%$ acabado) convencional producía un impacto de demanda de fósforo de 3,4 $\pm 0,36 \mathrm{~kg} \mathrm{P} / \mathrm{T}$.

En cuanto al impacto CED, destacan las fórmulas, según FEDNA, de la fase de lechones y acabado (4.236 $\mathrm{MJ} / \mathrm{T}$ pienso y $4.046 \mathrm{MJ} / \mathrm{T}$ pienso, respectivamente), próximas a los valores obtenidos por Garcia-Launay et al. (2016), de 4.442 $\pm 351,2 \mathrm{MJ} / \mathrm{T}$, formulando por objetivos económicos y ambientales. Para el impacto CED, valorado teniendo en cuenta el consumo de alimento anual, destaca la fase de acabado con 15.576 MJ/año y $8.844 \mathrm{MJ} /$ año, según FEDNA e ITAB, respectivamente.

En la valoración del GWP, destaca con mayor impacto la dieta de lechón (ITAB) y acabado (FEDNA), de $424 \mathrm{~kg} \mathrm{CO}_{2} / \mathrm{T}$ y $403 \mathrm{~kg} \mathrm{CO}_{2} / \mathrm{T}$, respectivamente, y el menor impacto del pienso de cerdas gestantes ITAB, con $275 \mathrm{~kg} \mathrm{CO}_{2} / \mathrm{T}$. En dietas estándar (con leguminosas, tortas de oleaginosas, cereales y sin aminoácidos de síntesis química), Reckmann et al. (2016) obtuvieron valores similares en crecimiento (452 $\mathrm{kg} \mathrm{CO}_{2} / \mathrm{T}$ pienso) y acabado $\left(479 \mathrm{~kg} \mathrm{CO}_{2} / \mathrm{T}\right)$, y superiores en gestantes $\left(467 \mathrm{~kg} \mathrm{CO}_{2} / \mathrm{T}\right)$. Formular $1 \mathrm{~kg}$ de pienso para lechones según las recomendaciones de ITAB produce un mayor impacto del potencial de cambio climático (GWP), comparado con FEDNA; debido a la utilización de un porcentaje superior de centeno y triticale ( $51 \%$ en total), que tienen un menor rendimiento estimado de la cose- 
Tabla III. Composición de los ácidos grasos de ingredientes ecológicos para piensos (\% sobre el total de ésteres metílicos de ácidos grasos detectados) (Composition of fatty acids of organic feed ingredients (\% on total methyl esters of fatty acids detected)).

\begin{tabular}{|c|c|c|c|c|c|c|}
\hline & AGS (\%) & AGMI (\%) & AGPI n-3 (\%) & AGPI n-6 (\%) & AGPI (\%) & AGPI/AGS \\
\hline \multicolumn{7}{|l|}{ Cereales } \\
\hline Avena & 19,54 & 38,16 & 2,01 & 40,27 & 42,28 & 2,17 \\
\hline Cebada & 27,36 & 14,71 & 6,45 & 51,48 & 57,92 & 2,45 \\
\hline Centeno & 17,97 & 14,15 & 11,04 & 56,84 & 67,88 & 3,78 \\
\hline Maíz grano & 14,44 & 27,63 & 1,68 & 56,26 & 57,93 & 4,08 \\
\hline Trigo & 19,93 & 15,71 & 4,99 & 59,40 & 64,39 & 3,24 \\
\hline Triticale & 18,01 & 13,67 & 7,13 & 61,19 & 68,32 & 3,82 \\
\hline \multicolumn{7}{|l|}{ Subproductos de cereales } \\
\hline Germen de maíz & 15,12 & 28,2 & 1,12 & 55,56 & 56,67 & 3,75 \\
\hline Harinilla de maíz & 13,4 & 25,36 & 1,69 & 59,55 & 61,24 & 4,57 \\
\hline Salvado de trigo & 21,14 & 16,58 & 4,76 & 57,54 & 62,3 & 2,95 \\
\hline \multicolumn{7}{|c|}{ Concentrados de proteína vegetal } \\
\hline Girasol entero & 12,24 & 28,52 & 0,08 & 59,17 & 59,25 & 4,84 \\
\hline Guisantes & 18,87 & 25,51 & 9,77 & 45,83 & 55,60 & 2,95 \\
\hline Alverjón & 17,73 & 31,27 & 7,67 & 43,34 & 51,00 & 2,88 \\
\hline Habas & 20,48 & 25,64 & 4,72 & 49,17 & 53,89 & 2,63 \\
\hline Soja entera & 13,63 & 19,27 & 10,21 & 56,89 & 67,10 & 4,94 \\
\hline Torta de presión de girasol & 14,44 & 44,1 & 0,36 & 41,11 & 41,47 & 2,87 \\
\hline Torta de presión de soja & 18,50 & 18,91 & 10,75 & 51,84 & 62,59 & 3,38 \\
\hline Yeros & 40,17 & 15,33 & 19,68 & 24,70 & 44,38 & 1,10 \\
\hline \multicolumn{7}{|l|}{ Ingredientes fibrosos } \\
\hline Cascarilla de espelta & 27,57 & 24,91 & 7,64 & 39,60 & 47,24 & 1,73 \\
\hline Granulado de alfalfa & 28,62 & 24,71 & 15,72 & 30,87 & 46,59 & 1,70 \\
\hline Paja de cereal & 48,52 & 10,69 & 14,44 & 25,92 & 40,35 & 0,90 \\
\hline \multicolumn{7}{|l|}{ Aceites } \\
\hline Aceite de soja & 13,44 & 25,80 & 8,42 & 52,35 & 60,77 & 4,53 \\
\hline
\end{tabular}

AGS: suma de ácidos grasos saturados (C10:0; C12:0; C14:0; C16:0; C17:0; C18:0; y C20:0); AGMI: suma de ácidos grasos monoinsaturados (C16:1n-7; C17:1n-7; C18:1n-9; y C20:1n-9). AGPI: suma de ácidos grasos poliinsaturados (AGPI: C18:2n-6; C18:3n-3; C20:2n-6; C20:3n-6; C20:4n-6; C20:4n-6 y C22:6n-3).

cha $(-20 \%$, comparado con cebada), lo que implica un mayor impacto del potencial de cambio climático por unidad de producto, de forma similar a otros estudios, como Müller et al. (2010) y Asseng et al. (2015). En este sentido, Espagnol et al. (2018) han observado que el impacto de cambio climático es inferior para leguminosas como el guisante o habas debido a su capacidad para fijar el nitrógeno atmosférico, lo que reduce de forma importante las necesidades de fertilización de suelos y las emisiones de $\mathrm{NO}_{2}$ asociadas. Según García-Launay et al. (2016), el impacto GWP de una dieta estándar mixta ( $40 \%$ crecimiento / $60 \%$ acabado) produce un impacto de $426 \pm 4,6 \mathrm{~kg} \mathrm{CO}_{2}$-eq/T de pienso.

Las fórmulas de acabado y lechones, según FED$\mathrm{NA}$, obtienen mayor impacto de $\mathrm{AP}$, con valores de 8 molc $\mathrm{H}^{+} \mathrm{eq} / \mathrm{T}$ de pienso producido, similares a los obtenidos por García-Launay et al. (2016), de 9,0 0 0,58 $\mathrm{mol} \mathrm{H}^{+} / \mathrm{T}$. Valorando el impacto por el consumo anual, resulta un 50\% superior la dieta de más impacto (aca- bado) según FEDNA en comparación con ITAB (30 vs. 20 molc $\mathrm{H}+\mathrm{eq}$, respectivamente).

En la valoración del impacto EP $\left(\mathrm{kg} \mathrm{PO}_{4} / \mathrm{T}\right)$, destacan las fases de lechones y acabado, con un $5,1 \mathrm{~kg}$ $\mathrm{PO}_{4} / \mathrm{T}$ y $4,5 \mathrm{~kg} \mathrm{PO} / \mathrm{T}$, según ITAB y FEDNA, respectivamente. El valor similar de EP entre dietas puede ser debido, como en el caso de la acidificación, a su estrecha relación con la aplicación de fertilizantes minerales, prohibidos en producción ecológica. Reckmann et al. (2016), en la fase de crecimiento, obtuvo valores inferiores, de $3,95 \mathrm{~kg} \mathrm{PO}_{4} / \mathrm{T}$, y en la fase de acabado, valores superiores, de 5,38 $\mathrm{kg} \mathrm{PO}_{4} / \mathrm{T}$.

El último impacto valorado ha sido la ocupación de tierra $\left(\mathrm{LO}, \mathrm{m}^{2}\right)$, dónde el valor más bajo se ha observado en la fase de acabado, con un valor de $1.748 \mathrm{~m}^{2} / \mathrm{T}$ (ITAB), próximo al resultado de García-Launay et al. (2016), de $1.418 \pm 59,5 \mathrm{~m}^{2}$. La dieta con mayor impacto es la de lechones, tanto en FEDNA como ITAB, de 2.310 y $2.171 \mathrm{~m}^{2} / \mathrm{T}$, respectivamente. Reckmann et al. (2016) 
Tabla IV. Ingredientes y contenido nutricional de dietas para porcino reproductor y engorde en ecológico ( $\%$ sobre materia fresca) ( Ingredients and nutritional content of diets for breeding pigs and organic fattening (\% on fresh matter)).

\begin{tabular}{|c|c|c|c|c|c|c|c|c|c|c|}
\hline \multirow[b]{2}{*}{ Ingredientes (\%) } & \multicolumn{2}{|c|}{ Lactantes } & \multicolumn{2}{|c|}{ Gestantes } & \multicolumn{2}{|c|}{ Lechones } & \multicolumn{2}{|c|}{ Crecimiento } & \multicolumn{2}{|c|}{ Acabado } \\
\hline & ITAB & FEDNA & ITAB & FEDNA & ITAB & FEDNA & ITAB & FEDNA & ITAB & FEDNA \\
\hline Avena & & & & 28,4 & & & & & & \\
\hline Cebada & & 31,7 & & & & & & & & \\
\hline Centeno & 24,0 & 12,0 & 30,6 & 10,0 & 22,1 & & 24,8 & 16,0 & 20,6 & 20,0 \\
\hline Maíz & & & & & & 29,5 & & 14,0 & & 26,9 \\
\hline Triticale & 25,4 & & 8,5 & & 28,5 & 9,3 & 26,1 & & 22,0 & \\
\hline Salvado de trigo & & & 35,0 & 28,9 & & & & & 13,6 & \\
\hline Guisantes & 18,1 & 15,4 & 20,0 & 16,0 & 15,1 & 10,0 & 15,0 & 20,0 & 14,8 & 17,6 \\
\hline Alverjón & 10,0 & 10 & 3,6 & 7,0 & & 10,0 & 10,0 & 18,5 & 9,4 & \\
\hline Alholva & 10,0 & 10 & & & & 5,0 & 10,0 & 2,5 & 10,0 & \\
\hline Habas & 9,8 & & 7,0 & 7,0 & & 5,0 & 11,2 & 10,5 & 6,9 & 10,0 \\
\hline Soja & & 9,9 & & & 15,0 & 15,0 & & 10,0 & & 10,0 \\
\hline Torta soja & & 5,7 & & & 16,2 & 11,1 & & 2,5 & & 11,0 \\
\hline Aceite soja & & 1,9 & & & & 1,8 & & 3,7 & & 2,0 \\
\hline Carbonato cálcico & 1,0 & 1,0 & 1,3 & 1,2 & 1,3 & 1,2 & 1,1 & 0,4 & 1,3 & 0,9 \\
\hline Fosfato bicálcico & 1,4 & 2,0 & 0,6 & 1,2 & 1,5 & 1,8 & 1,4 & 1,5 & 1,1 & 1,2 \\
\hline Corrector vitamínico-mineral & 0,4 & 0,4 & 0,4 & 0,4 & 0,4 & 0,4 & 0,4 & 0,4 & 0,4 & 0,4 \\
\hline \multicolumn{11}{|l|}{ Composición nutricional } \\
\hline Materia seca (\%) & 89,4 & 89,6 & 88,9 & 89,1 & 89,8 & 89,2 & 89,4 & 89,1 & 89,3 & 89,2 \\
\hline EN (MJ/kg) & 9,6 & 9,8 & 9,5 & 8,9 & 9,7 & 10,0 & 9,1 & 10,0 & 9,0 & 10,0 \\
\hline Proteína (\%) & 15,7 & 17,5 & 13,4 & 14,7 & 19,0 & 19,5 & 15,5 & 18,4 & 15,6 & 17,0 \\
\hline Lisina (Lys) (\%) & 0,8 & 1,0 & 0,6 & 0,7 & 0,9 & 1,0 & 0,8 & 1,0 & 0,8 & 0,9 \\
\hline Lys/EN & 0,86 & 0,97 & 0,63 & 0,79 & 0,96 & 1,00 & 0,89 & 1,04 & 0,85 & 0,91 \\
\hline Fibra Bruta (\%) & 6,0 & 7,6 & 5.9 & 8,7 & 5,0 & 5,0 & 6,0 & 6,7 & 6,2 & 5,6 \\
\hline FND (\%) & 18,1 & 19,8 & 21,7 & 26,1 & 11,4 & 15,0 & 18,0 & 16,0 & 19,9 & 15,3 \\
\hline EE $(\%)$ & 2,9 & 5,9 & 2,9 & 3,4 & 5,7 & 7,8 & 2,9 & 7,7 & 3,2 & 6,7 \\
\hline Almidón (\%) & 46,0 & 34,0 & 46,4 & 34,4 & 40,7 & 35,0 & 45,5 & 35,0 & 44,2 & 37,5 \\
\hline Calcio (\%) & 0,9 & 1,1 & 0,8 & 0,9 & 1,0 & 1,1 & 1,0 & 0,7 & 1,0 & 0,8 \\
\hline Fósforo (\%) & 0,6 & 0,7 & 0,5 & 0,6 & 0,6 & 0,6 & 0,6 & 0,6 & 0,6 & 0,5 \\
\hline AGPI/AGS & 3,39 & 2,82 & 3,26 & 2,77 & 3,71 & 3,67 & 3,40 & 3,34 & 3,40 & 3,58 \\
\hline
\end{tabular}

observó que en la fase de crecimiento requiere una ocupación de $1.400 \mathrm{~m}^{2} / \mathrm{T}$, en lactación de $1.440 \mathrm{~m}^{2} / \mathrm{T}$, en gestación de $1.210 \mathrm{~m}^{2} / \mathrm{T}$ y acabado de $1.510 \mathrm{~m}^{2} / \mathrm{T}$. Los sistemas ecológicos producen un menor rendimiento de los cultivos por superficie y eso requiere de mayor superficie que el sistema convencional (Müller et al., 2017).

La valoración de los diferentes impactos por el total de consumo anual es proporcional al consumo de pienso en las diferentes fases, siendo de menor a mayor (gestantes $<$ lactantes $<$ lechones $<$ crecimiento $<$ acabado). Früh et al. (2015) estimaron el consumo medio por cerdo de engorde en $300 \mathrm{~kg}$ y en cerda de $1.000 \mathrm{~kg} /$ año; en nuestro estudio los valores de consumo medio equivalen a $316,4 \mathrm{~kg}$ por cerdo engorde y en cerda de $1.150 \mathrm{~kg}$ al año.

Desde un punto de vista medioambiental, son más sostenibles las dietas formuladas según recomendaciones ITAB (Francia) que según recomendaciones FEDNA(España). Existe una relación inversamente proporcional entre la proximidad de las materias primas consumidas y el impacto ambiental de las mismas. Se desprende la necesidad de una materia prima proteica local para formular dietas en porcino ecológico en la fase de lechones, para contribuir a una verdadera producción ecológica, con bajo 
Tabla V. Ingredientes y contenido nutricional de las dietas de cerdas gestantes y de cerdos de acabado derivadas de la mezcla de un pienso núcleo de lactación y crecimiento, respectivamente, con ingredientes fibrosos (Ingredients and nutritional content of the diets of pregnant sows and finished pigs derived from the mixture of a core feed of lactation and growth, respectively, with fibrous ingredients).

\begin{tabular}{|c|c|c|}
\hline & & \\
\hline & GESTANTES & ACABADO \\
\hline Ingredientes (\%) & $\begin{array}{l}75 \% \text { pienso de } \\
\text { lactación } \\
+25 \% \text { alimen- } \\
\text { to fibroso }\end{array}$ & $\begin{array}{l}90 \% \text { pienso de creci- } \\
\text { miento } \\
+10 \% \text { subproductos ce- } \\
\text { reales }\end{array}$ \\
\hline Centeno & 18,0 & 22,0 \\
\hline Triticale & 19,0 & 23,5 \\
\hline Salvado trigo & 20,7 & 10,0 \\
\hline Paja de cereal & 4,9 & \\
\hline Guisantes & 13,5 & 13,5 \\
\hline Alverjón & 7,0 & 9,0 \\
\hline Alholva & 7,0 & 9,0 \\
\hline Habas & 7,0 & 10,0 \\
\hline Carbonato cálcico & 0,8 & 1,2 \\
\hline Fosfato bicálcico & 1,1 & 1,4 \\
\hline $\begin{array}{l}\text { Corrector } \\
\text { vitamínico-mineral }\end{array}$ & 0,4 & 0,4 \\
\hline $\begin{array}{l}\text { Composición nutric } \\
\mathrm{MJ} / \mathrm{kg} \text { ) de la mezcla }\end{array}$ & $\begin{array}{l}\text { al (\% sobre mate } \\
\text { e ingredientes fib }\end{array}$ & $\begin{array}{l}\text { ria fresca, excepto EN, en } \\
\text { cosos con el pienso núcleo }\end{array}$ \\
\hline Materia seca & 89,0 & 89,4 \\
\hline Energía & 9,1 & 9,0 \\
\hline Proteína & 14,0 & 15,5 \\
\hline Celulosa Bruta & 8,0 & 6,2 \\
\hline $\begin{array}{l}\text { Fibra } \\
\text { Neutro-detergente }\end{array}$ & 25,0 & 19,2 \\
\hline Extracto Etéreo & 3,2 & 2,7 \\
\hline Almidón & 41,1 & 39,4 \\
\hline Calcio & 0,8 & 1,0 \\
\hline Fósforo & 0,5 & 0,6 \\
\hline Lisina & 0,6 & 0,8 \\
\hline AGPI/AGS & 2,99 & 3,35 \\
\hline
\end{tabular}

impacto ambiental asociado, ya que la soja requerida no se produce actualmente en España. No obstante, existe posibilidad de cultivo de leguminosas (alfalfa, trébol, esparceta), que pueden podrían ser buenas alternativas para garantizar las necesidades proteicas en el resto de las fases de producción (gestantes, lactantes, crecimiento y acabado). Sin embargo, la utilización de estos forrajes requeriría la implantación de mezcladoras unifeed en las explotaciones que permitieran picar el forraje y mezclarlo con el pienso, o disponer de comederos adaptados para la oferta de pacas de forraje, como ya se hace en explotaciones de centro Europa (Rivera et al. 2001, Rivera et al. 2006,

\section{Wüstholz et al., 2017)}

Parte de la producción porcina ecológica (igual que la convencional) se basa en alimentación con piensos compuestos y mínimo uso de forrajes, y el aporte nitrogenado de las dietas deriva en gran parte de la soja y sus procesados, importados de Brasil, Italia o China, dónde el transporte en términos de huella de carbono (394 $\mathrm{g} \mathrm{CO}_{2}$-eq/ $\mathrm{kg}$ alimento) es aproximadamente el mismo que la huella de carbono por cultivo y procesado (326 g CO 2 -eq/ $\mathrm{kg}$ alimento) (Kristensen et al., 2011); además de la ocupación de territorio de la siembra de materias primas, en competición con los cultivos destinados a la producción humana (Hermansen et al, 2013).

La producción de piensos de porcino y aves contribuye de forma importante al impacto ambiental de la producción animal: representando el 50-85\% del impacto sobre el cambio climático, el $64-97 \%$ del potencial de eutrofización, el $70-96 \%$ de la demanda de energía y casi el $100 \%$ de la ocupación de tierra, variando según el tipo de sistema de producción y localización geográfica (Wilfart et al., 2016). La soja y sus derivados son incorporados en un rango de un $10-29 \%$ en la ración de porcino, el incremento del cual se ha visto favorecido por el descenso del precio, generando un impacto potencial importante en cuanto a cambio climático (Basset-Mens et al., 2005; Thoma et al., 2011; Nguyen et al., 2011; Dourmad et al., 2014). La dieta con mayor impacto ambiental es la de lechones y la de menor impacto es la de cerdas gestantes, dónde en energía renovable resultan valores de $4.236 \mathrm{MJ}$ vs. $1.971 \mathrm{MJ}$, respectivamente, en cambio climático $424 \mathrm{~kg}$ $\mathrm{CO}_{2}$ vs. $275 \mathrm{~kg} \mathrm{CO}_{2}$; acidificación de 7,7 molc $\mathrm{H}^{+}$-eq vs. 4,7 molc H+-eq; eutrofización 5,1 kg PO Ps. 3,2 kg PO $_{4}$ y ocupación de tierra $2.310 \mathrm{~m}^{2}$ vs. $1.349 \mathrm{~m}^{2}$ (Figura 1).

En la Tabla VI se resumen los resultados de los costes de compra de alimentos en fábrica de piensos y costes de autoproducción, según recomendaciones nutricionales ITAB, para una cerda reproductora y su descendencia durante un año (20 cerdos). Se observa que la compra de pienso resulta un $28 \%$ más cara, si se compara con un modelo de producción autosuficiente a nivel de finca. En la valoración económica de las dietas ecológicas, resultaría más rentable un modelo de negocio con integración agropecuaria, especialmente en las fases de cerdos de crecimiento y acabado, ya que es dónde se abaratan más los costes. Para la producción del alimento consumido (7.450 $\mathrm{kg}$ de pienso anuales) por una cerda reproductora y 20 cerdos de descendencia, se requiere una superficie necesaria de 2,55 ha de cultivo de cereales (1 ha) y leguminosa (1,5 ha).

\section{CONCLUSIONES}

Es posible formular dietas para porcino ecológico, utilizando como fuente proteica alverjón, habas, guisantes y alholva, y como fuente energética triticale, centeno, trigo y cebada, siguiendo las recomendaciones 



Figura 1. Comparación de la Representación de los impactos ambientales por Tn de alimento fabricado, según recomendación FEDNA e ITAB-IFIP y fase fisiológica: (a) cerda y (b) crecimiento-engorde (Comparison of the representation of environmental impacts by Tn of manufactured food, according to recommendation FEDNA and ITAB-IFIP and physiological phase: (a) sow and (b) growth-fattening).

nutricionales de ITAB, excepto en la fase de lechones $(<30 \mathrm{~kg})$.

En el modelo francés, la relación Lys/EN es menos exigente y se requiere una menor proporción de concentrado proteico vegetal ( $41 \%$ según ITAB vs. $52 \%$ según FEDNA) y más ingredientes fibrosos.

En la valoración ambiental de los impactos por unidad de pienso producido se observa que son supe-

Tabla VI. Costes de compra de pienso vs en comparación con la autoproducción en una granja porcina ecológica de ciclo cerrado produciendo 20 cerdos por cerda y año (Costs of buying feed vs compared to self-production in a closed-cycle organic swine farm producing 20 pigs per sow per year).

\begin{tabular}{lccc}
\hline Fase fisiológica & $\begin{array}{c}\text { Consumo } \\
\text { anual }(\mathrm{kg})\end{array}$ & $\begin{array}{c}\text { Compra } \\
\text { fábrica pien- } \\
\text { so* }\end{array}$ & $\begin{array}{c}\text { Autoproduc- } \\
\text { ción }\end{array}$ \\
$\begin{array}{l}\text { Gestante } \\
\text { Lactante }\end{array}$ & $\begin{array}{c}170 \\
\text { Lechón } \\
\text { (<30 kg; }\end{array}$ & $305,76 €$ & $107,52 €$ \\
$\begin{array}{l}\text { hasta 12 semanas } \\
\text { de edad) }\end{array}$ & 1.008 & $1.095,90 €$ & $943,88 €$ \\
$\begin{array}{l}\text { Crecimiento } \\
\text { (<60 kg; hasta 19 } \\
\text { semanas de edad) }\end{array}$ & 1.470 & $733,82 €$ & $328,64 €$ \\
$\begin{array}{l}\text { Acabado } \\
\text { (<110 kg; } \\
\text { hasta } 30 \text { semanas } \\
\text { de edad) }\end{array}$ & 3.850 & $1.686,30 €$ & $917,65 €$ \\
$\begin{array}{l}\text { Coste } \\
\text { de arrendamiento } \\
\text { de tierras }\end{array}$ & & & \\
\hline *Incluye transporte e impuestos & & \\
\hline
\end{tabular}

riores siguiendo las normas FEDNA que ITAB para la demanda de fósforo ( $\mathrm{kg} \mathrm{P})$, energía no renovable (MJ) y acidificación (molc $\mathrm{H}+\mathrm{eq}$ ).

Por el contrario, en el impacto por cambio climático $(\mathrm{kg} \mathrm{CO})$, eutroficación $\left(\mathrm{kg} \mathrm{PO}_{4}\right)$ y ocupación de tierra $\left(\mathrm{m}^{2}\right)$, las dietas de lechones y crecimiento tienen más impacto según recomendaciones del Instituto francés (ITAB). La dieta con mayor impacto ambiental es la de lechones y la de menor impacto la de cerdas gestantes.

\section{AGRADECIMIENTOS}

Arturo Salvador (Albalate de Cinca); Bernat Rosell (Cerdanya); Cal Pauet SCP (l'Espunyola); Celler Cooperatiu de Salelles; Cooperativa del Pallars (Sort); Ecofarm Trading SL; Ecològica dels Pirineus (Lleida); Joana Amador (Escola Agrària de Manresa); Fruits del Secà SCP (Sant Pere Sallavinera); Josep Argerich (Montmajor); Josep Bombardó (Cerdanya); Lluís Tor (Llívia); Martí Verdés (Gàver); Pep Tusón (Associació ERA); Pinsos Duranoc.

\section{BIBLIOGRAFIA}

Álvarez-Rodríguez, J, Ros-Freixedes, R, Gol, S, Henríquez-Rodríguez, E, Pena, RN, Bosch, L, Estany, J, Vilaró, F, \& Tor M 2018, 'Carcass lean-yield effects on the fatty acid and amino acid composition of Duroc pork and its technological quality after vacuum-aging ', Animal Production Science, vol. 58, no. 12, pp. 2335-2343.

AOAC (Association of Official Agricultural Chemists) 2000, Official Methods of Analysis, 17th ed. Association of Official Agricultural Chemists International, Arlington, VA, USA.Asseng, S, Ewert, F, Martre, P, Rötter R P, Lobell, D B, Cammarano, D, \& Reynolds, M P 2015, 'Rising temperatures reduce global wheat production', Nature Climate Change, vol. 5, no. 2, pp. 143.

Berry, PM, Sylvester-Bradley, R, Philipps, L, Hatch, DJ, Cuttle, SP, Rayns, FW, \& Goslin P 2002, 'Is the productivity of organic farms restricted 
by the supply available nitrogen?', Soil Use Management, vol. 18, pp 248-255.

Basset-Mens, C \& Van derWerf, HM 2005, 'Scenario-based environmental assessment of farming systems: the case of pig production in France', Agriculture, Ecosystems \& Environment, vol. 105, no. 1, pp. 127-144.

Berry, PM, Sylvester-Bradley, R, Philipps, L, Hatch, DJ, Cuttle, SP, Rayns, FW, \& Goslin, P 2002, 'Is the productivity of organic farms restricted by the supply available nitrogen?', Soil Use Management, vol. 18, pp. 248-255.

Calvar, C \& Maupertuis, F 2010, 'Porc biologique - quelques références techniques', Atout Porc Bretagne, Septembre 2010, pp. 12-15.

De Vries, M \& de Boer, I J 2010, 'Comparing environmental impacts for livestock products: A review of life cycle assessments', Livestock Science, vol. 128(1), pp. 1-11.

DOUE 2007, Reglamento (CE) 834/2007 del Consejo, de 28 de junio, sobre producción y etiquetado de los productos ecológicos y por el que se deroga el Reglamento (CEE) 2092/91, 23 pp.

DOUE 2008, Reglamento (CE) 889/2008 de la Comisión, de 5 de septiembre, por el que se establecen disposiciones de aplicación del Reglamento (CE) 834/2007 del Consejo sobre producción y etiquetado de los productos ecológicos, con respecto a la producción ecológica, su etiquetado y su control, $84 \mathrm{pp}$.

DOUE 2012, Reglamento de Ejecución (UE) 505/2012 de la Comisión, de 14 de junio, que modifica y corrige el Reglamento (CE) 889/2008, por el que se establecen disposiciones de aplicación del Reglamento (CE) 834/2007 del Consejo, sobre producción y etiquetado de los productos ecológicos, con respecto a la producción ecológica, su etiquetado y su control, $8 \mathrm{pp}$.

DOUE 2013, Reglamento (UE) 1306/2013 del Parlamento Europeo y del Consejo, de 17 de diciembre, sobre la financiación, gestión y seguimiento de la Política Agrícola Común, por el que se derogan los Reglamentos (CE) n ${ }^{\circ} 352 / 78$, (CE) n ${ }^{\circ} 165 / 94$, (CE) n ${ }^{\circ}$ 2799/98, (CE) n $814 / 2000$, (CE) n ${ }^{\circ} 1290 / 2005$ y (CE) n 485/2008 del Consejo, $59 \mathrm{pp}$.

DOUE 2018a, Reglamento (UE) 2018/848 del Parlamento Europeo y del Consejo, de 30 de mayo, sobre producción ecológica y etiquetado de los productos ecológicos y por el que se deroga el Reglamento (CE) n 834/2007 del Consejo, 92 pp.

DOUE 2018b, Reglamento de ejecución (UE) 2018/1584 de la Comisión de 22 de octubre de 2018 que modifica el Reglamento (CE) $n^{\circ} 889 / 2008$, por el que se establecen disposiciones de aplicación del Reglamento (CE) n 834/2007 del Consejo sobre producción y etiquetado de los productos ecológicos, con respecto a la producción ecológica, su etiquetado y su control, $12 \mathrm{pp}$.

Dourmad, J Y, Ryschawy, J, Trousson, T, Bonneau, M, Gonzàlez, J, Houwers H W J, \& Morgensen L 2014, 'Evaluating environmental impacts of contrasting pig farming systems with life cycle assessment', Animal, vol. 8, no. 12, pp. 2027-2037.

Edwards, S A 2003, 'Intake of nutrients from pasture by pigs', Proceedings of the Nutrition Society, vol. 62(2), pp. 257-265.

Espagnol, S, Tailleur, A, Dauguet, S, Garcia-Launay, F, Gaudré, D, Dusart, L, Méda, B, Gac, A, Laisse S, Morin, L, Dronne, Y, Ponchant, $P$, \& Wilfart, A 2018, 'Réduire les impacts environnementaux des produits animaux avec des éco-aliments', Innovations Agronomiques INRA, vol. 63, pp. 1-12.

FEDNA 2013, Necesidades nutricionales para ganado porcino, Normas FEDNA, 2nd ed. De Blas, C., Gasa, J., Mateos, G.G. (Eds.), Fundación Española para el Desarrollo de la Nutrición Animal, Madrid, España.
Früh, B, Schlatter, B, Isense,e A, Maurer, V, \& Willer, H 2015, 'Report on organic protein availability and demand in Europe', Research Institute of Organic Agriculture, Frick, Switzerland.

Früh, B 2016, 'Animal-friendly pig husbandry', Technical guide. Research Institute of Organic Agriculture (FiBL) and Sahee Foundation, $\mathrm{CH}-5070$ Frick.

Garcia-Launay, F, Wilfart, A, Dusart, L, Nzally, C, Gaudré, D, \& Espagnol $S 2016$, Multi-objective formulation is an efficient methodology to reduce environmental impacts of pig feeds, In 10th International Conference on Life Cycle Assessment of Food.

Garcia-Launay, F, Van der Werf, H M G, Nguyen, T T H, Le Tutour, L, \& Dourmad, J Y, 2014, 'Evaluation of the environmental implications of the incorporation of feed-use amino acids in pig production using Life Cycle Assessment', Livestock Science, vol. 161, pp. 158-175.

Gencat, 2017, 'Dades estadístiques de produccions agrícoles a Catalun$\mathrm{ya}^{\prime}$, Disponible en: http://agricultura.gencat.cat/ca/departament/ dar_estadistiques_observatoris/dar_estructura_produccio/dar_estadistiques_agricoles/dar_estadistiques_definitives

Gómez-Izquierdo, E G 2015, 'Efecto sobre el rendimiento productivo y calidad de la canal de la inclusión de guisantes (Pisum sativum) y alberión (Vicia narbonensis) en el pienso de lechones y cerdos de cebo' (Doctoral dissertation, Universidad Politécnica de Madrid).

Hermansen, J E, Knudsen, M T, \& Kristensen, T 2013, 'Role of animal products in feeding the planet. In Energy and protein metabolism and nutrition in sustainable animal production', Wageningen Academic Publishers, Wageningen, pp. 35-44.

INRA 2018, INRA feeding system for ruminants, Wageningen Academic Publishers.

ITAB 2014, 'Alimentation des porcins en agriculture biologique', Cahier technique, ITAB-IFIP-Chambre Agriculture Pays de la Loire.

Jakobsen, M \& Kongsted, A G 2014, 'Performance and behaviour of free-range pigs in relation to feed protein level and forage crop', In Book of Abstracts of the 65th Annual Meeting of the European Association for Animal Production Copenhagen, Denmark, 25-28 August 2014, Wageningen Academic Publishers.

Kristensen, T, Mogensen, L, Knudsen, M T, \& Hermansen, J E 2011, 'Effect of production system and farming strategy on greenhouse gas emissions from commercial dairy farms in a life cycle approach', Livestock Science, vol. 140, no. 1, pp. 136-148.

MAPAMA 2015, Observatorio de tecnologías probadas, Maquinaria agrícola, Disponible en: http://www.mapama.gob.es/es/ministerio/servicios/informacion/plataforma-de-conocimiento-para-elmedio-rural-y-pesquero/observatorio-de-tecnologias-probadas/ maquinaria-agricola/default.aspx (Consultado: 28 de enero de 2018).

MAPA 2016, 'Observatorio de tecnologías probadas', Maquinaria agrícola, Disponible en: https://www.mapa.gob.es/es/estadistica/ temas/estadisticas-agrarias/economia/canones-anuales-arrendamientos-rusticos/ (Consultado: 28 de enero de 2018)

Martín-Pedrosa, M, Varela, A, Guillamón, E, Cabellos, B, Burbano, C, Gómez-Fernández, J, \& Muzquiz, M 2016, 'Biochemical characterization of legume seeds as ingredients in animal feed', Spanish Journal of Agricultural Research', vol. 14(1), pp. 0901.

Müller, C \& Robertson, R D 2014, 'Projecting future crop productivity for global economic modeling', Agricultural Economics, vol. 45, no. (1), pp. 37-50.

National Research Council 2012, Nutrient requirements of swine, 11 th rev, National Academies Press, Washington, DC. 
Noblet, J, Valancogne, A, Tran, G, Ajinomoto Eurolysine S A S, EvaPig R 2008, Computer program. INRA, AFZ, Ajinomoto Eurolysine SAS, Saint-Gilles, Paris, France.

Reckmann, K, Blank, R, Traulsen, I, \& Krieter, J 2016, 'Comparative life cycle assessment (LCA) of pork using different protein sources in pig feed', Archives Animal Breeding, vol. 59, no. 1, pp. 27-36.

Rivera-Ferre, M G, Edwards, S A, Mayes R W, Riddoch I, \& Hovell F D 2001, 'The effect of season and level of concentrate on the voluntary intake and digestibility of herbage by outdoor sows', Animal Science, vol. 72, no. 3, pp. 501-510.

Rivera-Ferre, M G, Aguilera, J F, \& Nieto, R. 2006, 'Differences in whole-body protein turnover between Iberian and Landrace pigs fed adequate or lysine-deficient diets', Journal of animal science, vol. 84, no. 12, pp. 3346-3355.

Sabaté, P \& Solé, M 2003, 'Anàlisi de costos d'una explotació de cereal a la comarca de la Segarra', Departamentd'Administració d'Empreses i Gestió Econòmica dels Recursos Naturals de la Universitat de Lleida. Smith, J, Gerrard, CL, \& Hermansen, JE 2014, 'Improved Contribution of local feed to support $100 \%$ Organic feed supply to Pigs and Poultry'.
Tejido, M L, Ranilla, M J, Palacios, C, Saro, C, Sosa, A, Díaz, A, \& Carro, MD 2011, 'A comparison of the nutritive value of organically and conventionally grown barley and wheat crops', Opt. Med., A no. 99, Challenging strategies to promote the sheep and goat sector in the current global context, pp. 53-61.

Thoma, G, Nutter, D, Ulrich, R, Maxwell, C, Frank, J, \& East, C 2011 , 'National life cycle carbon footprint study for production of US swine', National Pork Board, Des Moines, IA.

Tor, M, Llovera, M, Moreno, JA, \& Angulo, E 2015, 'Evolución a lo largo del ciclo de puesta del contenido de ácidos grasos omega-3 en huevos enriquecidos de producción ecológica', Información técnica económica agraria-ITEA, vol. 111, no. 2, pp.142-153.

Wilfart, A, Espagnol, S, Dauguet, S, Tailleur, A, Gac, A, \& GarciaLaunay, F 2016, 'ECOALIM: A Dataset of Environmental Impacts of Feed Ingredients Used in French Animal Production', PloS one, vol. 11 , no. 12, e0167343.

Wüstholz, J, Carrasco, S, Berger, U, Sundrum, A, \& Bellof, G 2017, 'Fattening and slaughtering performance of growing pigs consuming high levels of alfalfa silage (Medicago sativa) in organic pig production', Livestock Science, vol. 200, pp. 46-52. 